\title{
LOS APÉNDICES INTERROGATIVOS EN EL CORPUS PRESEEA-SEVILLA: SOCIOLECTOS ALTO Y BAJO
}

\author{
Juana SANTANA MARRERO \\ Universidad de Sevilla
}

\section{RESUMEN}

En esta investigación nos centramos en la distribución sociolingüística de los apéndices interrogativos registrados en el corpus PRESEEA-Sevilla (sociolectos alto y bajo). Estos recursos le permiten al hablante asegurarse de que se mantiene abierto el contacto con el interlocutor y, al mismo tiempo, corroborar el consenso, el seguimiento o el conocimiento de la información a la que acompañan. Los resultados obtenidos revelaron que la forma más recurrente en la capital hispalense fue $i n o$ ?, la cual se empleó junto a otras unidades con una frecuencia intermedia, ¿eh?, ¿sabes?, ¿entiendes? y ¿vale?, o baja, ¿de acuerdo?, ¿um?, ¿verdad?, ¿ves?, ¿comprendes? y ¿me explico? El análisis sociolingüístico puso de manifiesto la influencia de los factores nivel educativo y edad en el reparto de estas partículas.

PALABRAS CLAVE: apéndices interrogativos, PRESEEA-Sevilla, sociolingüistica.

\section{AbSTRACT}

This investigation focuses on how the tag questions are distributed in PRESEEASeville corpus (high and low sociolects) from a sociolinguistic point of view. These mechanisms allow the speaker to make sure that the contact with the interlocutor remains open and, at the same time, to show agreement, to confirm following or knowledge of what it is being reported. The results showed that the most employed form in Seville was $i n o$ ?, along with other units which were halfused such as $\dot{i}$ eh? $\dot{i}$ sabes?, $\dot{i}$ entiendes? and $\dot{i}$ vale? , or on a low frequency like $\dot{i}$ de acuerdo?, ium?, iverdad?, ives?, icomprendes? and $\dot{i}$ me explico? The sociolinguistic analysis brought to light the influence that the educational level and age factors had over the distribution of these markers.

KEYWORDS: tag questions, PRESEEA-Seville, sociolinguistics. 


\section{Presentación ${ }^{1}$}

El estudio de los marcadores del discurso en español desde la perspectiva de la variación dialectal y social ha arrojado valiosos resultados en los últimos años (Carbonero y Santana 2010; Valencia 2014; Valencia y Vigueras 2015). Entre ellos cabe señalar los que se han llevado a cabo empleando los materiales de PRESEEA de diferentes ciudades del mundo hispánico como Madrid (Cestero 2019; Cestero y Moreno Fernández 2008; Molina 2006 y 2017), Medellín (Grajales Alzate 2011), México (Aldama y Reig 2016), Santiago de Chile (San Martín 2004-2005, 2011 y 2013; San Martín et alii 2016) o Sevilla (Santana 2015), entre otras. Se aúnan en estos trabajos las aportaciones del ámbito pragmático-discursivo, necesario para la caracterización adecuada de estas unidades, y las de la sociolingüística, perspectiva que permite conocer si se aprecian influencias de los rasgos sociales de los usuarios en su empleo. Dado el interés de los resultados alcanzados hasta el momento, en el XVIII congreso de ALFAL (Bogotá, julio de 2017), los equipos integrantes de PRESEEA asumieron el compromiso de incluir entre sus objetivos prioritarios el estudio coordinado de los apéndices interrogativos (Cestero 2003) o marcadores de control de contacto (Briz 2001; San Martín 2011). Esta investigación se enmarca dentro de dicha iniciativa, centrada en el corpus PRESEEA de la ciudad de Sevilla. Nos ocupamos de recursos del tipo ¿no?, ¿eh?, ¿vale?, ¿sabes?, ¿verdad?, entre otros. Para ello hemos utilizado los materiales de los sociolectos alto y bajo, compuestos por 48 grabaciones procedentes de encuestas semidirigidas, de unos $40 \mathrm{mi}-$ nutos cada una, recopiladas entre los años 2009 y 2017. El carácter dialogado del corpus favorece el análisis de los elementos bajo estudio. Siguiendo las premisas del Proyecto (Moreno Fernández 1996), la muestra está sometida a control sociolingüístico, incluyendo los parámetros de nivel educativo (alto: con estudios universitarios; bajo: sin estudios o con formación primaria), edad (primera generación: de 19 a 34 años; segunda generación: de 35 a 54 años; tercera generación: de 55 años en adelante) y sexo (distribución equilibrada entre hombres y mujeres) $^{2}$.

\footnotetext{
${ }^{1}$ Esta investigación se inserta dentro del proyecto Patrones sociolingüisticos del español de Sevilla (PASOS-SE) [Proyecto de Excelencia I+D+I, convocatoria de 2015, referencia FFI201568171-C5-3-P, financiado por el Ministerio de Economía y Competitividad de España y por el Fondo Europeo de Desarrollo Regional].

${ }^{2}$ Para la identificación de los ejemplos se emplea el siguiente sistema de codificación: en primer lugar se indica el sexo (H 'hombre' / M 'mujer'), a continuación el grupo de edad (1 'primera generación', 2 'segunda generación' y 3 'tercera generación') y seguidamente el nivel educativo (1 'bajo' y 3 'alto'). En último lugar se señala el orden que ocupa cada informante: del 001 al 024 para el sociolecto bajo y del 049 al 072 para el sociolecto alto.
} 
La finalidad última de esta investigación es conocer la rentabilidad que tienen las partículas seleccionadas en la ciudad hispalense: qué formas se emplean, con qué frecuencia y qué repercusión tienen los rasgos sociales de los hablantes en su distribución.

\section{CARACTERIZACión}

Los marcadores de los que aquí nos ocupamos conforman un grupo de unidades conversacionales que el hablante utiliza para reclamar la atención del interlocutor, con quien busca mantener el contacto, y cerciorarse de que el proceso comunicativo fluye sin problemas de comprensión o de seguimiento. Todos ellos tienen entonación interrogativa, aspecto que delata el ámbito de la interacción dialógica en el que actúan.

En lo que respecta a su forma, en unos casos están constituidos por una secuencia fonológica breve, a menudo monosilábica (ino?, ¿̨eh?, iah?, iya?), por lo que han sido considerados elementos «de cuerpo fónico reducido» (Ortega 1986: 272) o «microunidades» (Rodríguez Muñoz 2009: 84). En otros casos tienen origen en una unidad léxica con base en el campo semántico de 'la verdad' o 'la certeza' (¿verdad?, ¿cierto?) o en un verbo de 'percepción física o intelectual' (¿ves?, ¿sabes?). No es extraño encontrar algunas variaciones formales, habituales en los marcadores del discurso (Martín Zorraquino 2010: 104), debidas a diferencias dialectales (ientiendes? / ¿entendés?) o a que no ha culminado plenamente el proceso de gramaticalización (¿comprendes? / ¿comprende?; ¿ves? / ¿tú ves?).

La caracterización pragmático-discursiva que se ha hecho de estas partículas en la bibliografía especializada y las diferentes denominaciones que han recibido dan cuenta de las principales facetas que las definen: funciones fática y apelativa. Briz (2001: 224-225) ubica estas unidades dentro de los marcadores de control de contacto, en tanto que «implican activamente al interlocutor» en el proceso comunicativo y lo hacen partícipe de «razonamientos de los hablantes». San Martín (2011: 139) se suma a esta denominación y considera que son partículas «mediante las cuales los hablantes mantienen la atención y controlan la comprensión del resto de los participantes en un intercambio comunicativo». Por su parte, Cestero (2002: 620) resalta también su carácter fático, pues sirven para «asegurarse de que el canal de comunicación está abierto y funciona, es decir, confirmar que se mantiene la atención y el seguimiento continuos». En lo que respecta a la faceta exhortativa, Fuentes (1990: 172) incluyó parte de estos marcadores dentro de los apéndices con valor apelativo que, en palabras de la autora, «tienen como función apelar al oyente, llamar su atención, pedir su colabo- 
ración en el acto discursivo». En Fuentes y Brenes (2014: 186) se insiste también en este carácter apelativo como el «valor básico» de estas unidades, a través de las cuales «el emisor le solicita al receptor que se comporte como tal, que preste atención a lo dicho». Tanto Cortés y Camacho (2005: 165-177), quienes caracterizan formas del tipo $\dot{i}$ me explico?, $i v e r d a d ?$ o $_{i}$ comprendes? como "marcadores interactivos centrados en el oyente», como Galué (2002: 36), para quien estos elementos «son especialmente utilizados para interpelar al interlocutor y atraer su atención», se decantan también por la apelación como su cualidad más sobresaliente.

A la función básica que les hemos atribuido, marcas que actúan en el intercambio dialógico con las que el hablante se asegura de que se mantiene el contacto con el interlocutor y de que la comunicación fluye con éxito, hay que sumarles valores o matices más específicos. Estos dependen de la intencionalidad puntual con la que se han empleado y en ocasiones están influidos por la base léxico-semántica de la forma que dio origen al marcador. Ambos aspectos nos permitirán perfilar con más precisión qué finalidad se persigue al contactar e interpelar al interlocutor. Aunque no es nuestro objetivo tratar con detenimiento este aspecto, proponemos una aproximación inicial a las principales funciones que se aprecian en nuestro corpus $^{3}$. Para ello seguimos de cerca la tipología propuesta por Cestero (2003: 87-96).

- Búsqueda de Ratificación. Este es uno de los rasgos más característicos de estas partículas, de ahí que Martín Zorraquino y Portolés Lázaro (1999: 4188) las denominen apéndices comprobativos. En unos casos la corroboración va dirigida hacia el contenido al que acompaña el marcador. Es lo que Cestero (2003: 89) llama «búsqueda de ratificación de acierto». El hablante requiere la complicidad del oyente para constatar que comparte con él la información que está aportando. Se trata de lo que Móccero (2010: 71) delimita como "petición de confirmación de hechos». García Vizcaíno (2005: 91-92), en su análisis de $\dot{i} n o$ ?, señala que este proceso de constatación admite la paráfrasis por «¿no es así?». En ocasiones, incluso, el interlocutor reacciona verbalmente a este requerimiento y da una respuesta.

(1) I: la de <alargamiento/> El Cristo de los Gitanos // sabes cuál es įno? // E: sí (H13-050)

En otros casos la búsqueda de consenso no va dirigida a confirmar que el contenido aportado se ajusta a la realidad y al cono-

\footnotetext{
${ }^{3}$ Está en fase de desarrollo aplicar a nuestra muestra una delimitación más precisa de los papeles pragmático-discursivos que cumplen estas unidades en los diferentes contextos en los que se emplean y su posible distribución sociolingüística (Cestero 2019).
} 
cimiento compartido por ambos actores del diálogo, sino que se solicita acuerdo sobre una opinión expresada. Es lo que Cestero (2003: 88) identifica como «búsqueda de ratificación de acuerdo» y Móccero (2010: 71) como «petición de evaluación». El hablante trata de cerciorarse de que su interlocutor es de su mismo parecer. Para estos casos, García Vizcaíno (2005: 91-92) propone la paráfrasis por «¿no crees?» o «¿no estás de acuerdo?».

(2) con ese el <vacilación/> ese ruedo / con ese albero tan bonito que le da tanto colorido ¿verdad? a la plaza (M33-069)

Marcadores como $\underset{i}{n o}$ ? o $\dot{i} v e r d a d$ ? se emplean a menudo con la función pragmático-discursiva de ratificación de contenido o de opinión, según se ha visto en (1) y (2) respectivamente.

- Comprobación de la CONTINUIDAD de la inTERACCión. La finalidad del marcador aquí es mantener abierta la comunicación con el oyente, al que alude en su discurso, pero sin la intención específica de buscar una reacción en él. Como apuntan Fuentes y Brenes (2014: 186), la función apelativa característica de estos apéndices interrogativos pierde intensidad hasta «derivar hacia la simple constatación del desarrollo del proceso de comunicación, llegando a convertirse en elementos fáticos». García Vizcaíno (2005: 92) señala para estos casos la posible paráfrasis por «¿me sigues?». Este valor se da especialmente cuando el marcador se convierte en una estrategia recurrente en la intervención de un sujeto y constituye un apoyo discursivo que favorece el avance de la información, como se aprecia en (3).

(3) la Feria de noche prácticamente / hay algunas casetas que más que casetas parecen discotecas o sea que / o < vacilación/ $>$ o simplemente bares de copas ¿̨no? más que lo que es una debe o debería de ser una caseta ¿no? el Rocío / pues el Rocío también está $<$ alargamiento/> ipff! muy cambiado / además ha cambiado a una velocidad tremenda en muy poco tiempo ino? ya cada vez mmm los accesos por el campo son más complicados (H23-053)

- Constatación de entendimiento o de COnOcimiento. La intención del hablante ahora es reclamar la atención del interlocutor para que le preste especial atención a una información y, al mismo tiempo, le corrobore que la entiende o que la conoce, a fin de evitar malentendidos. A este respecto, Ortega (1986: 275) le atribuía una significación bastante cercana al sentido literal del verbo saber: tratar de «deshacer la ignorancia» del oyente. Este contenido está asociado especialmente a aquellas partículas que tienen origen en formas verbales del campo semántico de la 'percepción intelectual' como ientiendes?, ¿comprendes? o isabes? 
(4) y entonces pues / con quince años / aparte de que en la casa hacía falta el dinero // E: <simultáneo $>$ sí $</$ simultáneo $>/ /$ I: $<$ simultáneo $>$ ¿comprendes? </simultáneo $>$ (H31-012)

- Enfatización. La misión de la partícula aquí es reclamar la atención del oyente para destacar un contenido con la finalidad de que no le pase desapercibido. Esto es lo que García Vizcaíno (2005: 94) y Rodríguez Muñoz (2009: 94) llaman «refuerzo expresivo", función que ambos autores comprueban que es muy rentable en el caso de $\dot{i}$ eh?

(5) y me dice / quillo Paco este número me suena a mí įeh? (H31011)

Los valores señalados no son necesariamente excluyentes, sino que pueden superponerse y combinarse en una misma partícula.

\section{ANÁLISIS DE LA MUESTRA}

En este apartado vamos a estudiar el comportamiento y la rentabilidad de los marcadores interrogativos de interacción conversacional empleados por los informantes sevillanos encuestados. Para ello dividiremos nuestros resultados en dos bloques: en uno primero aportaremos una visión global del rendimiento y la distribución sociolingüística de todas estas partículas en nuestro corpus, y en uno segundo nos detendremos de forma individualizada en los recursos específicos localizados en la muestra. Por las limitaciones de espacio con las que contamos, en esta segunda parte nos centraremos en resaltar aquellos aspectos que aporten resultados relevantes para la investigación.

\subsection{Análisis global}

El corpus arrojó un total de 1311 apéndices interrogativos, repartidos en 11 formas distintas.

En esta primera aproximación se aprecia el claro predominio de $\dot{i} n o$ ?, el cual se revela como el apéndice prototípico entre los encuestados (857/ $1311,65,37 \%)$. A ello hay que sumar que fue empleado por la mayoría de los informantes $(44 / 48,91,67 \%)$, generalmente como el elemento con mayor rentabilidad en cada uno de ellos (31/44, 70,45\%). En consonancia con estos resultados, los datos de la norma culta hispánica revelan también que fue la forma más empleada a uno y otro lado del Atlántico (Santana 2017: 244-246). No solo se registraron casos en todas las ciudades sondeadas, sino que en la mayoría de ellas fue además el recurso más utilizado. 
GRÁFICO 1. Distribución global

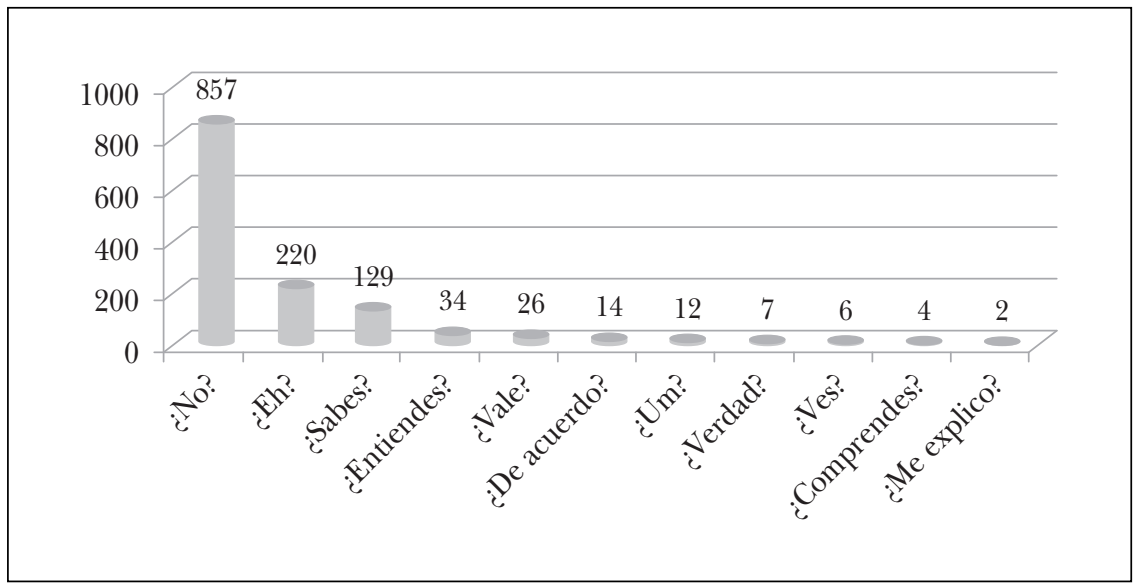

En el lado opuesto de la escala se sitúan ¿de acuerdo?, ¿um?, ¿verdad?, ¿ves?, ¿comprendes? y ime explico? que, con menos de 15 ocurrencias, relegadas a unos pocos informantes, son marcas poco extendidas entre los sujetos analizados.

Tabla 1. Distribución según sociolecto

\begin{tabular}{|c|c|c|c|c|}
\hline & \multicolumn{2}{|c|}{ Alto } & \multicolumn{2}{|c|}{ Bajo } \\
\hline & $\mathrm{n}$ & $\%$ & $\mathrm{n}$ & $\%$ \\
\hline ¿No? & 644 & 75,15 & 213 & 24,85 \\
\hline ¿Eh? & 111 & 50,45 & 109 & 49,55 \\
\hline ¿Sabes? & 58 & 44,96 & 71 & 55,04 \\
\hline ¿Entiendes? & 2 & 5,88 & 32 & 94,12 \\
\hline ¿Vale? & 5 & 19,23 & 21 & 80,77 \\
\hline ¿De acuerdo? & 14 & 100,00 & 0 & 0,00 \\
\hline ¿Um? & 12 & 12,00 & 0 & 0,00 \\
\hline ¿Verdad? & 5 & 71,43 & 2 & 28,57 \\
\hline ¿Ves? & 2 & 33,33 & 4 & 66,67 \\
\hline ¿Comprendes? & 1 & 25,00 & 3 & 75,00 \\
\hline ¿Me explico? & 0 & 0,00 & 2 & 100,00 \\
\hline TOTAL & 854 & 65,14 & 457 & 34,86 \\
\hline
\end{tabular}


TABLA 2. Distribución según edad

\begin{tabular}{|c|c|c|c|c|c|c|}
\hline & \multicolumn{2}{|c|}{$1^{a}$ generación } & \multicolumn{2}{|c|}{$2^{\mathrm{a}}$ generación } & \multicolumn{2}{|c|}{$3^{\mathrm{a}}$ generación } \\
\hline & $\mathrm{n}$ & $\%$ & $\mathrm{n}$ & $\%$ & $\mathrm{n}$ & $\%$ \\
\hline$\dot{\zeta} N o ?$ & 136 & 15,87 & 392 & 45,74 & 329 & 38,39 \\
\hline ¿Eh? & 25 & 11,36 & 77 & 35,00 & 118 & 53,64 \\
\hline ¿Sabes? & 83 & 64,34 & 35 & 27,13 & 11 & 8,53 \\
\hline ¿Entiendes? & 1 & 2,94 & 10 & 29,41 & 23 & 67,65 \\
\hline ¿Vale? & 14 & 53,85 & 10 & 38,46 & 2 & 7,69 \\
\hline ¿De acuerdo? & 0 & 0,00 & 14 & 100,00 & 0 & 0,00 \\
\hline$\dot{\zeta} U m ?$ & 0 & 0,00 & 3 & 25,00 & 9 & 75,00 \\
\hline ¿Verdad? & 0 & 0,00 & 3 & 42,86 & 4 & 57,14 \\
\hline ¿Ves? & 1 & 16,67 & 3 & 50,00 & 2 & 3,33 \\
\hline ¿Comprendes? & 0 & 0,00 & 1 & 25,00 & 3 & 75,00 \\
\hline ¿Me explico? & 0 & 0,00 & 0 & 0,00 & 2 & 100,00 \\
\hline TOTAL & 260 & 19,83 & 548 & 41,80 & 503 & 38,37 \\
\hline
\end{tabular}

Tabla 3. Distribución según sexo

\begin{tabular}{|c|c|c|c|c|}
\hline & \multicolumn{2}{|c|}{ Hombres } & \multicolumn{2}{|c|}{ Mujeres } \\
\hline & $\mathrm{n}$ & $\%$ & $\mathrm{n}$ & $\%$ \\
\hline$\dot{i} N o$ ? & 532 & 62,08 & 325 & 37,92 \\
\hline ¿Eh? & 120 & 54,55 & 100 & 45,45 \\
\hline ¿Sabes? & 60 & 46,51 & 69 & 53,49 \\
\hline ¿Entiendes? & 5 & 14,71 & 29 & 85,29 \\
\hline ¿Vale? & 12 & 46,15 & 14 & 53,85 \\
\hline ¿De acuerdo? & 14 & 100,00 & 0 & 0,00 \\
\hline ¿Um? & 0 & 0,00 & 12 & 100,00 \\
\hline ¿Verdad? & 1 & 14,29 & 6 & 85,71 \\
\hline ¿Ves? & 4 & 66,67 & 2 & 33,33 \\
\hline ¿Comprendes? & 4 & 100,00 & 0 & 0,00 \\
\hline ¿Me explico? & 0 & 0,00 & 2 & 100,00 \\
\hline TOTAL & 752 & 57,36 & 559 & 42,64 \\
\hline
\end{tabular}


Por nivel educacional, los individuos del sociolecto alto emplearon más frecuentemente estos marcadores. Aun así, fueron los hablantes con baja formación académica los que presentaron mayor tendencia al empleo de elementos distintos. Si bien lo más habitual fue la combinación de 2 o 3 formas diferentes en un mismo informante $(28 / 48,53,33 \%)$, esta media subió entre los hablantes con nivel educativo bajo, donde se concentró la mayor parte de los sujetos que utilizaron 4 (5/7, 71,43\%), $5(2 / 2,100,00 \%)$ y $6(2 / 3,66,67 \%)$ apéndices interrogativos distintos.

La edad puso de manifiesto que, de forma general, estos marcadores fueron menos habituales en las intervenciones de los encuestados más jóvenes. La variable sexo, por su parte, mostró un ligero incremento entre los hombres.

Los datos que arroja nuestra muestra presentan amplias coincidencias con las del corpus PRESEEA-Madrid (Cestero 2019: 43-44), por lo que habría que comprobar si estamos ante patrones sociolingüísticos más generalizados.

\subsection{Análisis por marcador}

A continuación centraremos nuestra atención en las particularidades más destacables del comportamiento de los apéndices interrogativos localizados en el corpus. De forma general cabe decir que a menudo estos elementos se convierten en un rasgo idiolectal de algunos hablantes: en el caso de las formas más recurrentes, hay sujetos que concentran en sus intervenciones índices de frecuencia superiores a la media; en el caso de las unidades menos rentables, a menudo los registros se concentran en unos pocos individuos. Según veremos, las características sociales de estos informantes influyen en la distribución sociolingüística de cada recurso ${ }^{4}$.

\subsection{1 ¿No?}

Como ya se ha apuntado, es el marcador interrogativo más característico de la muestra $(857 / 1311,65,37 \%)$. Se empleó a menudo para la búsqueda de ratificación, según se ve en (6), y para la comprobación de la continuidad de la interacción, como se advierte en (7).

(6) el negocio no se trata del dinero se trata de la distracción / ¿no? // E: <simultáneo> sí </simultáneo> (H33-057)

\footnotetext{
${ }^{4}$ Para los siguientes apartados nos basamos en los datos presentados en las tablas 1, 2 y 3 .
} 
(7) ¿lo que más me gusta de mi casa? / mmm < silencio/> no sé / me gusta entera la casa / ¿no? pero eh lo que más me gusta de la casa es que no tengo vecinos arriba ni abajo $<$ risas $=" \mathrm{E} " />/$ que es una casa $\dot{i} n o$ ? he vivido siempre en casa $\dot{i} n o$ ? y la verdad que cuando me metí en un piso / pues bueno / era lo que había ¿no? no se podía otra cosa // y luego con el tiempo pues al final / vinimos a una casa $\underset{i}{n}$ ? (H23-055)

La función puramente fática, apelar al interlocutor para mantener abierta la comunicación, es la que sobresale en aquellos contextos en los que el apéndice se emplea de forma recurrente. Esta característica fue especialmente llamativa en 3 varones del sociolecto alto: H23-053 (78 casos), H23055 (110 casos) y H33-059 (94 ejemplos).

Es importante recordar el valor de atenuación que se ha asociado a $i_{i} n o$ ? (Albelda y Cestero 2011: 33-34; Cestero y Albelda 2012: 96). En efecto, la búsqueda de complicidad del tú (bien para ratificar la certeza de lo que se ha dicho o de la opinión expresada, o bien para mantener el contacto con el oyente) se puede convertir en una estrategia que protege la imagen del hablante ante posibles reproches o réplicas. Hacer al interlocutor partícipe de nuestro discurso nos da cierta garantía de que aquello que decimos va a resultar menos impositivo, especialmente cuando se trata de opiniones controvertidas. Así se aprecia en el ejemplo (8), donde se está defendiendo la diversión en un entorno de religiosidad, la peregrinación del Rocío, tesis que cuenta con muchos detractores.

(8) realmente hay gente muy devota / y porque $<$ secuencia inacabada $>$ la mayoría / y < vacilación/ $>$ y también lo toman como una fiesta / porque yo creo que <alargamiento/> es motivo de fiesta ¿̨no? / tú sales con tus amigos a una $<$ vacilación/ $>$ una romería / a una pere $<$ palabra_corta$\mathrm{da} />\mathrm{a}$ una peregrinación / y <alargamiento/> y ¿por qué tienes que ir / por narices $<$ alargamiento/ $>/$ serio? $($ H13-049)

En nuestro corpus $\dot{i} n o$ ? fue un recurso más empleado por los informantes con mayor nivel de instrucción $(644 / 857,75,15 \%)$, por el segundo grupo etario $(392 / 857,45,74 \%)$ y por los hombres $(532 / 857,62,08 \%)$. Los varones fueron también los principales impulsores de esta estrategia en Madrid y en Valencia (Cestero 2019: 45; Cestero y Albelda 2012: 96). Se observa, además, que en Sevilla los índices de frecuencia descienden significativamente conforme baja la edad de los informantes. A pesar de que fue un marcador utilizado por la mayoría de los encuestados, la caracterización social de los sujetos que lo emplearon de manera recurrente incide en los índices de frecuencia de los sectores a los que pertenecen (veíamos que eran hombres del sociolecto alto, de la segunda y tercera generación). Si ponemos en relación nuestros datos con la función de atenuación, los hombres buscaron más la complicidad del interlocutor, fueron a este respecto más conciliadores, reclamando más a menudo su consenso. 


\subsection{2 ¿Eh?}

Este es el segundo marcador más empleado en nuestros materiales (220/ $1311,16,68 \%$ ), aunque a bastante distancia de ino? También está menos extendido entre el total de los individuos sondeados $(32 / 48,66,67 \%)$. Un valor habitual en esta partícula es el de enfatización. El contacto con el interlocutor va encaminado a hacerle notar la relevancia del segmento al que acompaña el apéndice interrogativo. Incluso se aprecian a menudo matices modales como advertencia, como se ve en (9), o amenaza.

(9) pero te voy a decir una cosa i $e h$ ? dentro de que pronuncian muy bien / hay cosas que yo <alargamiento/> no las entiendo (M31-023)

En lo que respecta a la correlación con las variables sociales, $\dot{\iota} e h$ ? fue un recurso algo más abundante entre los encuestados de la tercera generación $(118 / 220,53,64 \%)$ y entre los varones $(120 / 220,54,55 \%)$. Más específicamente, las mujeres del tercer grupo etario fueron las principales impulsoras de esta partícula $(70 / 220,31,82 \%)$. Nuestros datos se ven favorecidos por la recurrencia en su uso, superior a la media, en un hombre y una mujer de la segunda y la tercera generación respectivamente (H21-006, 40 casos y M33-069, 34 casos).

Consideramos un dato destacado que en nuestra muestra $\dot{\zeta} e h$ ? es un marcador que tiende a ser poco utilizado entre los jóvenes.

\subsection{3 ¿Sabes?}

Este elemento tiene su origen en un verbo de percepción intelectual, lo que determina su principal función: reclamar la atención del interlocutor para que corrobore que ha entendido o que conoce del contenido al que acompaña. A ello puede ir sumado un valor de enfatización, de realce informativo del segmento sobre el que se incide (Fuentes 2009: 310; Molina 2017).

(10) sí y se paliaría el cambio climático / porque / la <vacilación > la vegetación retendría gran parte del carbono isabes? / en Cuba / que tampoco es un modelo de país (H13-050)

El análisis del corpus PRESEEA-Sevilla puso de manifiesto que este recurso constituye una marca de estratificación social. En este sentido, a pesar de ser la tercera forma más empleada (129/1311, 9,84\%), su uso fue registrado fundamentalmente en el sociolecto bajo $(71 / 129,55,04 \%)$ y en hablantes jóvenes $(83 / 129,64,34 \%)$.

Nuestros resultados, además, tienen puntos de coincidencia con los obtenidos por Molina (2006 y 2017) en los barrios de Salamanca y de Vallecas, 
en la ciudad de Madrid. La autora demostró que a mayor nivel sociocultural son las mujeres las que más promueven el uso, mientras que cuando hay menor grado de instrucción la partícula está fundamentalmente anclada en el discurso de los hombres. En las encuestas de Sevilla, al contrastar los rasgos nivel educativo y sexo, se demuestra que son las féminas del sociolecto alto $(39 / 129,67,24 \%)$ y los varones del bajo $(41 / 129,57,75 \%)$ quienes prefieren el empleo de esta unidad, siempre dentro de los dos primeros grupos etarios ${ }^{5}$. Estos datos están condicionados por su concentración en una mujer y un hombre de la primera generación con grado de instrucción bajo (M13-061 y H21-006). Se trata, por tanto, de un marcador más asentado entre los jóvenes, que goza de cierto prestigio cuando es promovido por las mujeres con estudios universitarios.

\subsection{4 ¿Entiendes? ¿comprendes?}

Estos dos apéndices interrogativos se originan también en unidades verbales del campo de la 'percepción intelectual'. Su papel fundamental en el discurso guarda relación con ese origen semántico: constatación de entendimiento. Es decir, se apela al interlocutor para que le corrobore al hablante la comprensión del contenido expresado.

(11) lo que he vivido es el mundo gallego nunca me puedo meter en el mundo tropical / ientiendes? / porque <alargamiento/ $>$ / eh yo no he vivido el mundo tropical (H33-057)

(12) tienes que hacer cosas estructurales / coyunturales / que no sean cosas de $<$ vacilación/ > de un día / ¿comprendes? / y además que atraiga a gente $<$ vacilación/> gente buena (H23-056)

En nuestro corpus estos dos elementos no solo registraron una frecuencia absoluta poco representativa $(34 / 1311,2,59 \% ; 4 / 1311,0,31 \%)$, sino que muestran también tendencia al progresivo desuso, dado que sus registros van decreciendo con la edad de los informantes. También fueron recursos más destacados en los informantes con bajo grado de instrucción $(32 / 34,94,12 \% ; 3 / 4,75,00 \%)$. A este respecto, la mayoría de los casos de $\dot{i}$ entiendes? se concentran en una mujer de la tercera generación del sociolecto bajo (M31-023).

${ }^{5}$ Uniendo ambos niveles educativos, los encuestados de mayor edad constituyeron el grupo menos numeroso $(11 / 129,8,53 \%)$. 


\section{2 .5 ¿Vale?}

Una función característica de esta partícula en nuestro corpus es la de corroborar el contacto con el interlocutor. Se trata de una marca que a menudo sirvió para que el hablante se asegurase de que su interlocutor estaba atento, de que seguía abierta la comunicación. A este valor hay que añadir el de enfatización del segmento al que acompaña el marcador, que el hablante presenta como una información relevante.

(13) no cobro / porque no soy funcionaria <risas= "todos" $/>$ pero estoy trabajando en un comedor escolar $\dot{i} v a l e$ ? llevo muchos años también (M11-016)

El papel puramente fático sobresale en aquellos informantes que tienen este apéndice interrogativo como marca idiolectal, como fue el caso de una mujer joven del sociolecto bajo (M11-016). Este dato guarda relación con la correlación de ¿vale? con las variables sociales: fue un recurso más habitual entre los sujetos de menor nivel educacional $(21 / 26,80,77 \%) \mathrm{y}$, fundamentalmente, entre los hablantes de los dos primeros grupos etarios (24/26, $92,31 \%$ ). Si contrastamos este último dato con el hecho de que no registramos ningún ejemplo en las encuestas del habla de Sevilla de los años 70 y 80 (Santana en prensa), podemos considerar que es una marca conversacional cuyo uso se ha ido extendiendo en esta comunidad de habla en las últimas décadas.

\subsection{6 ¿De acuerdo? y ¿um?}

A pesar de la escasa frecuencia registrada, estas partículas se perfilaron en nuestro corpus como marcas de estratificación diastrática, en tanto que solo se documentaron usos entre los informantes con mayor grado de instrucción y no se dio ningún caso entre los sujetos más jóvenes. El dato del nivel educativo nos parece relevante si tenemos en cuenta que Cestero (2003: 110 y 121) comprobó que son elementos habituales en el discurso académico, contexto que se acerca más al perfil de un hablante culto.

De hecho, ¿ de acuerdo? fue un marcador que solo se registró en un informante varón que se dedica a la docencia (H23-056), principalmente para comprobar el mantenimiento de la interacción.

(14) he estado también de < vacilación / / eh / en jefatura de estudios / ¿de acuerdo? / y / eh <alargamiento/ $>$ / me he dedicado / especialmente en verano / a recuperar a niños que psi < palabra_cortada/> psicológicamente (H23-056) 
Lo mismo cabe decir para $\dot{\zeta} u m$ ?, empleado preferentemente por una mujer (M33-069) cuya labor profesional se desarrolla también en el ámbito de la enseñanza $(9 / 12,75 \%)$.

(15) se mudaron a Los Remedios / que entonces empezaba // y desde entonces vivo en Los Remedios / ¿ um? de soltera y de casada (M33-069)

\subsection{7 ¿Verdad?}

En último lugar nos fijamos en un recurso que tiene una base léxica en el campo semántico de 'la certeza' o 'lo verdadero'. Está en sinonimia discursiva con otras marcas de origen semántico similar icierto? / ¿no es cierto?, cuyos usos en la norma culta solo se registraron en Hispanoamérica (Santana 2017: 253-254). Los valores más destacados de ¿verdad? en nuestra muestra fueron los de ratificación, como se ve en (16), y de comprobación de la continuidad de la interacción, según aparece en (17).

(16) pues mientras más me divierta lo que estoy haciendo y menos mire la hora / más feliz seré / ¿verdad? (H33-057)

(17) muchísima ilusión porque claro / ya estaba un poquito $<$ risas = "I" $/>$ harta de colegios de monjas / ¿verdad? y me hacía mucha ilusión irme a una academia (M33-069)

Los datos obtenidos revelan su escasa rentabilidad entre los hábitos lingüísticos de los encuestados $(7 / 1311,0,53 \%)$. Esa baja frecuencia, unida a la falta de casos entre los informantes más jóvenes, hacen pensar en la tendencia al progresivo descenso de su uso en el habla de la ciudad hispalense.

\section{Conclusiones}

El estudio de los apéndices interrogativos en el corpus PRESEEA de la ciudad de Sevilla pone de manifiesto la amplia rentabilidad de estas partículas en la lengua hablada. La fórmula prototípica es $i n o$ ?, con elevados índices de uso y con registros en la mayoría de los encuestados. El resto de las unidades presentan una frecuencia que podríamos dividir entre media (ieh?, ¿sabes?, ¿entiendes?, ¿vale?) y baja (ide acuerdo?, ¿um?, ¿verdad?, ¿ves?, ¿comprendes?, ¿me explico?). El análisis cuantitativo arrojó algunos indicios de estratificación social.

El factor nivel educacional nos permitió comprobar que ¿de acuerdo? y $\dot{\zeta} u m$ ? fueron solo documentados en el sociolecto alto, en consonancia con su habitual presencia en el discurso académico. Por otro lado, la forma 
¿vale? fue empleada preferentemente por hablantes del sociolecto bajo. La edad de los informantes aportó datos interesantes acerca de algunas partículas que, al apenas documentarse ejemplos entre los más jóvenes, puede preverse la tendencia a su desaparición de los hábitos lingüísticos de los sevillanos. Fue el caso de ientiendes?, ¿de acuerdo?, ¿um?, ¿verdad? y icomprendes? Por su parte, $\dot{i} n o$ ? y $\dot{\zeta} e h$ ? experimentaron un descenso significativo entre los sujetos de menor edad. En el otro extremo hemos de señalar la aparición de recursos que van poco a poco asentándose entre la forma de hablar de los primeros grupos etarios, como sucedió con ¿sabes? y ¿vale? Podríamos estar ante un cambio generacional en el que las partículas más clásicas o tradicionales van siendo sustituidas por otras más "modernas" que se van extendiendo entre las generaciones más jóvenes. El sexo de los informantes nos permitió comprobar que el marcador $\dot{i} n o$ ?, en ocasiones asociado a la función de atenuación, fue más empleado por los hombres.

Está en nuestro ánimo seguir avanzando en el análisis de estos apéndices interrogativos y de otros marcadores del discurso en el corpus PRESEEASevilla para profundizar en las funciones pragmáticas específicas que cumplen en el discurso y estudiar su posible distribución sociolingüística. 


\section{BIBLIOGRAFÍA}

Albelda, Marta y Ana María Cestero (2011): «De nuevo sobre los procedimientos de atenuación lingüística», Español Actual 96, 11-42.

Aldama, Juan Diego y Asela ReIg (2016): «Variación sociolingüística en el empleo de un nuevo marcador discursivo: "ahora sí que" en el español de México", Boletín de Filología L1/2, 15-47.

BRIZ, Antonio (2001): El español coloquial en la conversación: esbozo de pragmagramática, Barcelona: Ariel Lingüística.

Carbonero, Pedro y Juana Santana (2010): «Los marcadores y la variación espacial y social». En Ó. Loureda y E. Acín (coords.), La investigación sobre marcadores del discurso del español, hoy, Madrid: Arco/Libros, 497-521.

Cestero, Ana María (2002): «La función fática del lenguaje en el discurso y en la conversación». En M. D. Muñoz et alii (eds.), Actas del IV Congreso de Lingüística General, Cádiz: Universidad de Cádiz, 617-629.

- (2003): «El funcionamiento de los apéndices interrogativos en la conversación y en el discurso académico». En C. Castillo y J. M. Lucía (eds.), Decíamos ayer... Estudios de alumnos en honor a María Cruz García de Enterría, Alcalá de Henares: Universidad de Alcalá, 83-127.

- (2019): «Apéndices interrogativos de control de contacto: estudio sociolingüístico», Cuadernos de Lingüística de El Colegio de México 6/1, 1-65.

- y Francisco Moreno Fernández (2008): «Usos y funciones de vale y ivenga! en la ciudad de Madrid», Boletín de Lingüistica 20. <http://www.scielo.org.ve/ scielo.php?pid=S0798-97092008000100003\&script=sci_arttext $>$.

- y Marta Albelda (2012): «La atenuación lingüística como fenómeno variable», Oralia $15,77-124$.

CORTÉs, Luis y María Matilde CAMACHO (2005): Unidades de segmentación y marcadores del discurso, Madrid: Arco/Libros.

Fuentes, Catalina (1990): «Apéndices con función apelativa». En P. Carbonero (dir.) y T. Palet (ed.), Habla de Sevilla y hablas americanas (= Sociolingüistica Andaluza 5), Sevilla: Publicaciones de la Universidad de Sevilla, 171-196.

- (2009): Diccionario de conectores y operadores del español, Madrid: Arco/Libros.

- y Ester BREnes (2014): «Apéndices apelativos en el lenguaje parlamentario andaluz: variación pragmática», Oralia 17, 181-209.

GALuÉ, Dexy (2002): «Marcadores conversacionales: un análisis pragmático», Boletín de Lingüistica 18, 27-48.

García Vizcaíno, María José (2005): «El uso de los apéndices modalizadores ¿̨no? y $\dot{b}_{e h}$ ? en español peninsular». En L. Sayahi y M. Westmoreland (eds.), Selected Proceedings of the Second Workshop on Spanish Sociolinguistics, Somerville, MA: Cascadilla Proceedings Project, 89-101. 
Grajales Alzate, Róbinson (2011): «Funciones del marcador discursivo pues en el habla de Medellín, Colombia», Forma y función 24/1. <http://www.scielo.org.co/ scielo.php?script=sci_arttext\&pid=S0120-338X2011000100002>.

Martín Zorraquino, María Antonia (2010): «Los marcadores del discurso y su morfología». En Ó. Loureda y E. Acín (coords.), La investigación sobre marcadores del discurso del español, hoy, Madrid: Arco/Libros, 93-181.

- y José Portolés LÁzAro (1999): «Los marcadores del discurso». En I. Bosque y V. Demonte (eds.), Gramática descriptiva de la lengua española, Madrid, EspasaCalpe, vol. 3, 4051-4421.

MócCERo, Leticia (2010): «Las preguntas confirmatorias como indicadoras de posicionamiento intersubjetivo», Estudios Filológicos 45, 67-78.

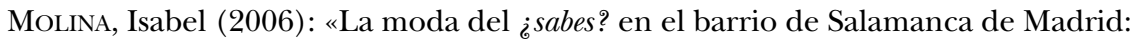
un análisis sociolingüístico». En Filología y lingüística. Estudios ofrecidos a Antonio Quilis, Madrid: CSIC/UNED/Universidad de Valladolid, I, 1045-1056.

- (2017): «El apéndice interrogativo ¿sabes? y su doble difusión en la estructura social de la periferia de Madrid (Vallecas)», Lingüistica en la Red XV. Procesos de variación y cambio en el español de España. Estudios sobre el corpus PRESEEA. $<$ http://www.linred.com>.

Moreno Fernández, Francisco (1996): «Metodología del 'Proyecto para el Estudio Sociolingüístico del Español de España y de América' (PRESEEA)», Lingüistica 8, 257-287.

ORTEGA, Jenaro (1986): «Aproximación al mecanismo de la conversación», Verba 13, 269-290.

RodRíGUEZ MuÑoz, Francisco (2009): «Estudio sobre las funciones pragmadiscursivas de ¿̇no? y ïeh? en el español hablado», Revista de Lingüística Teórica y Aplicada 47/1, I Sem., 83-101.

SAn MARTín, Abelardo (2004-2005): «Igual como marcador discursivo en el habla de Santiago de Chile: función pragmático-discursiva y estratificación social de su empleo», Boletín de Filología de la Universidad de Chile XL, 201-232.

- (2011): «Los marcadores interrogativos de control de contacto en el corpus PRESEEA de Santiago de Chile», Boletín de Filología de la Universidad de Chile XLVI/2, 135-166.

- (2013): «Los reformuladores de distanciamiento en el corpus PRESEEA de Santiago de Chile», Boletín de Filología de la Universidad de Chile XLVIII/1, 171-199.

—, Cristian Rojas Inostroza y Silvana Guerrero (2016): «La función discursiva y la distribución social de los marcadores por ser y onda en el corpus del PRESEEA de Santiago de Chile», Boletín de Filología de la Universidad de Chile LI/2, 235-254.

SANTANA, Juana (2015): «Marcadores del discurso y variación diastrática: análisis en el sociolecto bajo». En J. Santana (dir.) y M. León Castro y A. Zerva (eds.), La variación en el español actual. Estudios dedicados al profesor Pedro Carbonero (= Sociolingüistica Andaluza, 17), Sevilla: Editorial Universidad de Sevilla, 289-313.

- (2017): «Marcadores interrogativos de interacción conversacional en la norma culta hispánica». En 90 años de la Academia Boliviana de la Lengua, La Paz: Academia Boliviana de la Lengua, 232-286.

- (en prensa): "Apéndices interrogativos en el habla de Sevilla: un estudio en tiempo real», Itinerarios 29. 
VAlENCIA, Alba (coord.) (2014): Marcadores discursivos en la norma culta hispánica: 1964-2014, Cuadernos de la ALFAL 5. <http://www.mundoalfal.org/?q=es/con tent/cuadernos-de-la-alfal-n\%C2\%BA5>.

- y Alejandra Vigueras (coords.) (2015): Marcadores hispánicos. Usos de España y América en el corpus de estudio de la norma culta, México: Universidad Nacional Autónoma de México. 